\title{
Alpha proteobacterial ancestry of the [Fe-Fe]-hydrogenases in anaerobic eukaryotes
}

\author{
Mauro Degli Esposti ${ }^{1,2 *}$, Diego Cortez ${ }^{2}$, Luis Lozano², Simon Rasmussen ${ }^{3}$, Henrik Bjørn Nielsen ${ }^{3}$ \\ and Esperanza Martinez Romero²
}

\begin{abstract}
Eukaryogenesis, a major transition in evolution of life, originated from the symbiogenic fusion of an archaea with a metabolically versatile bacterium. By general consensus, the latter organism belonged to a proteobacteria, subsequently evolving into the mitochondrial organelle of our cells. The consensus is based upon genetic and metabolic similarities between mitochondria and aerobic a proteobacteria but fails to explain the origin of several enzymes found in the mitochondria-derived organelles of anaerobic eukaryotes such as Trichomonas and Entamoeba. These enzymes are thought to derive from bacterial lineages other than a proteobacteria, e.g., Clostridium - an obligate anaerobe. [FeFe]-hydrogenase constitues the characteristic enzyme of this anaerobic metabolism and is present in different types also in Entamoeba and other anaerobic eukaryotes. Here we show that a proteobacteria derived from metagenomic studies possess both the cytosolic and organellar type of [FeFe]-hydrogenase, as well as all the proteins required for hydrogenase maturation. These organisms are related to cultivated members of the Rhodospirillales order previously suggested to be close relatives of mitochondrial ancestors. For the first time, our evidence supports an a proteobacterial ancestry for both the anaerobic and the aerobic metabolism of eukaryotes. Reviewers: This article was reviewed by William Martin and Nick Lane, both suggested by the Authors.
\end{abstract}

Keywords: Eukaryogenesis, Anaerobic metabolism, [FeFe]-hydrogenase, Mitochondria, Bioenergetics

\section{Background}

Mounting interest is arising on eukaryogenesis, a major transition in evolution which most likely originated from a symbiogenic event between an archaea and a metabolically versatile bacterium $[1,2]$. The archaean partner of this symbiogenic event appears to be related to the Loki organisms recently discovered in deep ocean vents [2]. In contrast, the definition of the bacterial lineage for the other prokaryotic partner of eukaryogenesis remains unclear and controversial [2-9]. Recent analysis of major bioenergetic systems shared by bacteria and mitochondria suggests that a subset of extant $\alpha$ proteobacteria could be related to the ancestral lineage from which mitochondrial organelles of eukaryotic cells evolved $[4,8,10]$. However, biochemical and phylogenetic evidence

\footnotetext{
* Correspondence: mauro.degliesposti@iit.it; mauro1italia@gmail.com ${ }^{1}$ Italian Institute of Technology, Via Morego 30, 16136 Genoa, Italy ${ }^{2}$ Center for Genomic Sciences, UNAM Cuernavaca, Cuernavaca, Mexico Full list of author information is available at the end of the article
}

emerged from the study of anaerobic eukaryotes seriously challenges the possibility that $\alpha$ proteobacterial organisms may be the ancestors also for the anaerobic metabolism of eukaryotes such as Trichomonas and Entamoeba [11, 12].

Trichomonas, Entamoeba and other eukaryotes adapted to anaerobic conditions possess Mitochondria Related Organelles (MRO [3, 12-14]) carrying out reactions of anaerobic metabolism that are typical of obligate anaerobes such as Clostridium, rather than $\alpha$ proteobacteria [12-19]. [FeFe]-hydrogenase constitutes the distinctive enzyme of this anaerobic metabolism and is present in different types [12-19], both in MRO and the cytosol of anaerobic eukaryotes such as Mastigamoeba [18]. The wide molecular variations of $[\mathrm{FeFe}]$-hydrogenases $[11,12,15-19]$ are present in many obligate anaerobes, in particular Clostridiales, Thermotogales and $\delta$ proteobacteria [12-19]; only a few facultative anaerobic $\alpha$ proteobacteria such as Rhodospirillum rubrum have similar 
hydrogenases, but not the maturases required for their biogenesis [13]. Consequently, it has been concluded that the eukaryotic types of this enzyme have been laterally acquired from bacterial lineages other than $\alpha$ proteobacteria, along various elements of anaerobic metabolism [11-13, 16-19]. Clearly, this conclusion undermines the hypothesis that a single $\alpha$ proteobacterium could have been the ancestor of both the anaerobic and the anaerobic metabolism of eukaryotes $[1,3,6,8]$.

Here we show that some $\alpha$ proteobacterial organisms found in metagenomic analysis of human microbiota [20] possess the two major types of eukaryotic [FeFe]hydrogenases, as well as all genes needed for its assembly and maturation. Moreover, we report that relatives of photosynthetic Rhodospirillum [21] and methanotrophic $\alpha$ proteobacterial previously indicated as possible relatives of the mitochondrial ancestors $[10,22]$ possess a major type of eukaryotic $[\mathrm{FeFe}]$-hydrogenases that could be the ancestor also of the hydrogenase-related Nar1 protein, which is involved in the biogenesis of Fe-S clusters of all eukaryotes [23]. Our findings thus rectify previous conclusions, strengthening the possibility that eukaryotes inherited also their anaerobic metabolism from a single $\alpha$ proteobacterial ancestor.

\section{Results and discussion}

Some a proteobacteria possess all the maturases for [FeFe]-hydrogenase

[FeFe]-hydrogenase requires the concerted action of three maturases, called HydDEF [13, 19, 24], for the complex assembly of its active site, the $\mathrm{H}$ cluster (see [25-27] for most recent advances on the subject). Previous studies concluded that the [FeFe]-hydrogenases present in $\alpha$ proteobacterial organisms such Rhodospirillum rubrum are probably inactive or play a different role because the genome of such organisms does not contain the $H y d D E F$ genes for maturases [13, 19]. However, we have found that bacteria closely related to $R$. rubrum possess all Hyd maturases and multiple genes for [FeFe]-hydrogenases (Table 1 and Additional file 1: Figure S1). These organisms include two species of the genera Phaeospirillum [21] and also Pararhodospirillum [28] (previously Rhodospirillum photometricum [29]). Two bacteria of the $\mathrm{N}_{2}$ fixing genus of Pleomorphomonas, classified among the Methylocystaceae family of methanotrophs [30], also possess the same set of proteins (Table 1). Moreover, we have found the presence of Hyd maturases in proteobacterial organisms of metagenomic origin that are classified among the $\alpha$ proteobacterial order of Rhodospirillales (Table 1), which have been assembled directly from the human gut microbiome [20]. The genes of these organisms were assembled from clustering of more than 396 human gut samples representing dense clusters and assembled to high quality draft genomes; their taxonomic classification was found to be robust at the genus level [20]. These clusters were confirmed and refined using a large collection of 2309 metagenomics samples. Given the read coverage and the quality of the assemblies, we expect the genomes to be nearly complete, with low probability that they may include genes from other sources [20]. In particular, Acetobacter sp. CAG:977 was identified in 14 samples and represented by as much as $4.5 \%$ of the sequence reads in a stool sample while Azospirillum sp. CAG:239 was represented by as much as $11.6 \%$ of the sequence reads in another stool sample. Other Rhodospirillales of human gut metagenomic origin containing Hyd maturases are Azospirillum sp. CAG:260 (Table 1) and Acetobacter sp. CAG:267 (see Additional file 1: Figure S1). Their proteins lie either in upstream or sister position with respect to the majority of the homologous maturase proteins of eukaryotes (see Additional file 1: Figure S1).

We found that one gene for the long form of [FeFe]hydrogenase in Acetobacter CAG:977 and Azospirillum sp. CAG:239 is associated with genes coding homologues of two redox subunits of respiratory complex I, which form the NADH-reacting module of the enzyme: NuoE and NuoF (Table 1, cf. [31-34]). The eukaryotic orthologs of these proteins are the 24 and $51 \mathrm{kDa}$ subunits of mitochondrial complex I that are always coded by nuclear DNA, while the rest of complex I subunits is encoded by mtDNA in several protists $[5,32,34]$. The same proteins are found in the hydrogenosomes - a specialised form of MRO [19] of Trichomonas [11] and Sawyeria, an anaerobic Heterolobosea [35], which lack complex I [19]. The gene cluster containing the long form of [FeFe]-hydrogenase and NuoEF homologues in the organisms identified from metagenomic analysis [20] resembles the hym $A B C$ operon described in Clostridiales [36, 37] (Table 1). In turn, this operon clearly derives from the Fds operon of NAD-dependent formate dehydrogenase [38], from which the NADH-reacting module of respiratory complex I originated [32, 34]. Of note, the genome of the abovementioned metagenomic $\alpha$ proteobacteria invariably contains the gene for Pyruvate:Ferredoxin Oxidoreductase (PFO, Table 1), a key enzyme in the anaerobic metabolism of Clostridiales [36, 37] and also anaerobic eukaryotes [19]. To summarise, we report the first example of $\alpha$ proteobacterial organisms that have all the $H y d$ maturases required for the assembly and function of [FeFe]-hydrogenase enzymes (Table 1), which often are present with multiple forms in their genome (Table 1).

\section{Uncultivated a proteobacteria possess both major forms of eukaryotic [FeFe]-hydrogenases}

Anaerobic eukaryotes share diverse types of [FeFe]-hydrogenase with bacteria $[11-19,39]$. The longest form 
Table 1 Proteins of anaerobic metabolism in cultivated and uncultivated a proteobacteria

\begin{tabular}{|c|c|c|c|c|c|c|c|c|c|}
\hline \multirow{3}{*}{$\begin{array}{l}\text { Taxonomic group } \\
\text { Genome }\end{array}$} & \multicolumn{9}{|c|}{ Accession number of indicated proteins } \\
\hline & \multirow{2}{*}{$\begin{array}{l}\text { Clostridiales } \\
\text { full genome }\end{array}$} & \multicolumn{6}{|l|}{ a proteobacteria } & \multicolumn{2}{|l|}{ Eukaryotes } \\
\hline & & full genome & full genome & full genome & metagenome & metagenome & $\begin{array}{l}\text { metagenome assembly, } \\
\text { refined }\end{array}$ & full genome & full genome \\
\hline Organism & $\begin{array}{l}\text { Eubacterium } \\
\text { acidaminophilum }\end{array}$ & $\begin{array}{l}\text { Pleomorphomonas } \\
\text { oryzae }\end{array}$ & $\begin{array}{l}\text { Phaeospirillum } \\
\text { molischianum }\end{array}$ & $\begin{array}{l}\text { Pararhodospirillum } \\
\text { photometricum }\end{array}$ & $\begin{array}{l}\text { Azospirillum } \\
\text { CAG:260 }\end{array}$ & $\begin{array}{l}\text { Azospirillum } \\
\text { CAG:239 }\end{array}$ & $\begin{array}{l}\text { Acetobacter MGS:igc08677, } \\
\text { formerly CAG:977 }\end{array}$ & $\begin{array}{l}\text { Entamoeba } \\
\text { invadens }\end{array}$ & $\begin{array}{l}\text { Trichomonas } \\
\text { vaginalis }\end{array}$ \\
\hline \multicolumn{10}{|l|}{ Protein } \\
\hline Fdsy/NuoE-like & CAC39229, HymA & WP_051228661 & CCG42368, HoxS & $\begin{array}{l}\text { WP_041793924, } \\
\text { HoxS }\end{array}$ & $?$ & CDB53275 & $\begin{array}{l}\text { MH0030_GL0011535, } \\
\text { CCZ22624 }\end{array}$ & absent & AAV65813 \\
\hline Fds $\beta /$ NuoF-like & CAC39230, HymB & WP_026791755 & & & $?$ & CDB53276 & $\begin{array}{l}\text { MH0276_GL0125578, } \\
\text { CCZ22625 }\end{array}$ & absent & AAV65812 \\
\hline $\begin{array}{l}\text { Fdsa/NuoG-like, type M3 } \\
\text { [FeFe]-hydrogenase }\end{array}$ & CAC39231, HymC & $\begin{array}{l}\text { WP_026791754, } \\
\text { WP_036838588 }\end{array}$ & $\begin{array}{l}\text { CCG42367, } \\
\text { CCG40656 }\end{array}$ & WP_051013609 & $?$ & CDB53277 & $\begin{array}{l}\text { MH0030_GL0047537, } \\
\text { CCZ22626 }\end{array}$ & $\begin{array}{l}\text { M2 } \\
\text { derivatives }\end{array}$ & $\begin{array}{l}\mathrm{M} 2 \& \mathrm{M} 3 \\
\text { derivatives }\end{array}$ \\
\hline type A [FeFe]-hydrogenase & AHM56319 & absent & absent & absent & CDB39301 & CDB53824 & $\begin{array}{l}\text { MH0143_GL0097789, } \\
\text { CCZ21919 }\end{array}$ & Yes, 5 & absent \\
\hline HydE maturase & WP_025434761 & WP_051228492 & WP_002729849 & WP_041796462 & CDB39229 & CDB54134 & $\begin{array}{l}\text { MH0030_GL0015854, } \\
\text { CCZ22110 }\end{array}$ & absent & Yes, $\geq 2$ \\
\hline HydF maturase & WP_025434763 & WP_026790854 & WP_002729850 & WP_041793928 & CDB40806 & CDB53483 & $\begin{array}{l}\text { MH0030_GL0007678, } \\
\text { CCZ21461 }\end{array}$ & absent & Yes, $\geq 2$ \\
\hline HydG maturase & WP_025434762 & WP_026790852 & WP_002729851 & WP_041793926 & CDB40807 & CDB54364 & $\begin{array}{l}\text { MH0030_GL0045387, } \\
\text { CCZ22171 }\end{array}$ & absent & Yes, $\geq 2$ \\
\hline PFO reductase & AHM55439 & WP_036840581 & CCG40004 & CCG09362 & CDB40895 & $?$ & $\begin{array}{l}\text { MH0143_GL0088070, } \\
\text { CCZ21865 }\end{array}$ & Yes, $\geq 5$ & Yes, $\geq 1$ \\
\hline Short ferredoxin & WP_025436274 & WP_026790610. & CCG39997 & CCG06940 & CDB40908 & CDB53367 & $\begin{array}{l}\text { MH0030_GL0000056, } \\
\text { CCZ22365 }\end{array}$ & Yes, $\geq 2$ & Yes, $\geq 3$ \\
\hline
\end{tabular}

apdated Meta Genomic Species with current CCZ accession number of formerly CAG:977

?not present in currently incomplete genome 
of the enzyme, type M3 [12], is also the most common [40] and appears to be the progenitor of diverse eukaryotic variants [14]. At the $\mathrm{N}$-terminus, type 3 proteins share three FeS domains with the NuoG subunit of complex I $[11,12]$. Other types of [FeFe]-hydrogenase derive from the M3 type by differential loss of these FeS domains and progressive relaxation of sequence conservation in the Small Subunit (SSU) domain at the C-terminus [41], as summarised in the scheme of Fig. 1a. The cultivated a proteobacteria which we found to possess all Hyd

A
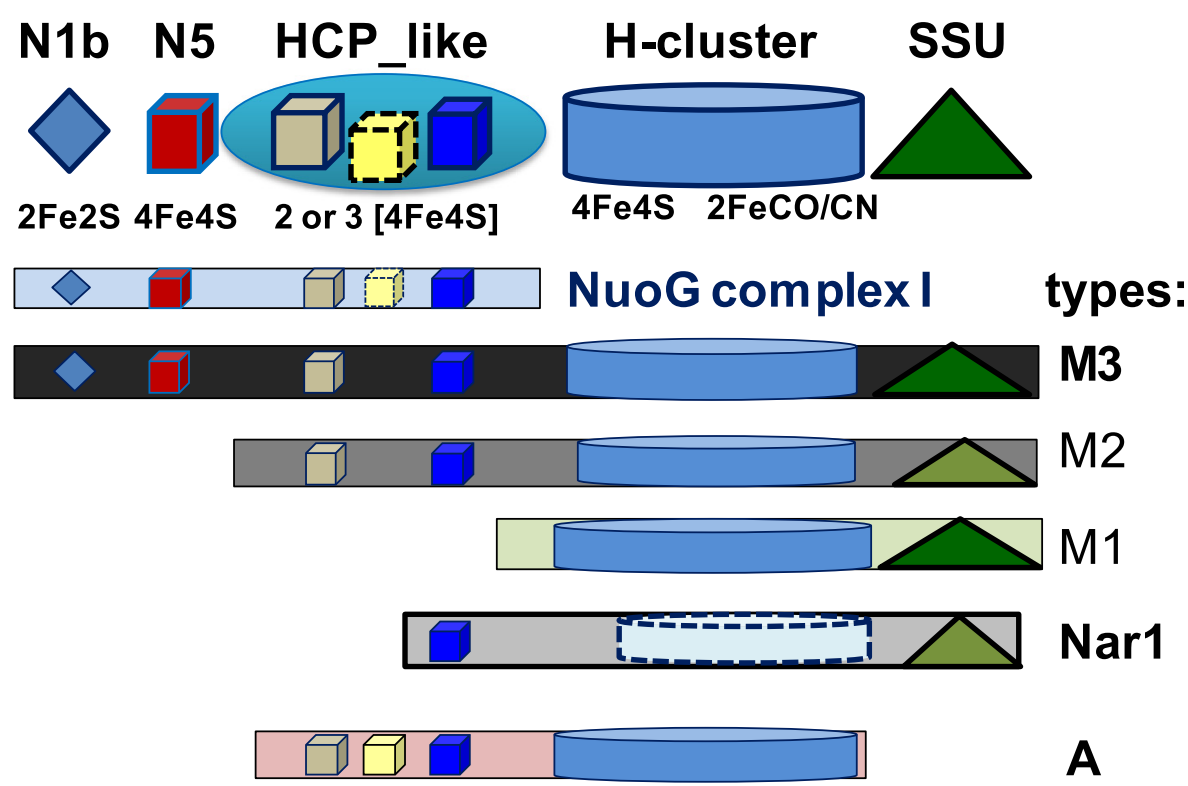

\section{B Alignment of $\mathrm{N}$-terminus of [FeFe]-hydrogenases}

\begin{tabular}{|c|c|}
\hline ession & cluster 1 HCP \\
\hline WP 043744126 & ¿QHCDQP PCMEVCPSTATRKRDDGIVTIDYDICIGCSYCAVSCP \\
\hline $\mathrm{CAG} 37108$ & EVTNACQGCLAQACIQSCPKDAITMVQ-GKSHIDSNLCINCGKCLKVCP \\
\hline CCZ30335 & IDVCRGCFAQPCRLNCPKEAISIVN-GRARIDPDKCVNCGKCFEVCP \\
\hline CDA18058 & EVTPMCQACVARPCETNCAKKAITVTDKAFIEQDK--CIKCGLCATYCP \\
\hline 18 & FVTNACQGCVARPCMSTCPKKAIS-RVDGQAKIDPDLCVRCGACQKVCP \\
\hline 66 & RVTNMCEGCTARPCYTNCPKKCIDFDENGQARIDQNICIH \\
\hline 820 & 'VTQACEGCTSRPCSVNCPKKCISFGEDGRAVINQNNCIKCGRCYKFCP \\
\hline 919 & KIDPEKCKSCGRCKEACPYQAI---------MR---CI--VPCEEACP \\
\hline 824 & CHEVCPYHSI---------RIP-----VPCEEACP \\
\hline 388063 & VTIDPELCTGCGRCVETCP---VQAIAGS----------RGKA------ \\
\hline & ELCTTKC--T----EDV-----LEKG-K \\
\hline 06 & ISADWSRCLGCNLCTTKC--T----FDV-----LKKE-AVKIPPF---- \\
\hline 27 & SCNMCIKAC--D-VQGIGV-----YKQNEKPKYPPI---- \\
\hline 433 & IMIDFQKCVGCNMCVKAC--VEQQKIGV-----LKAG-PPRSPPYR--- \\
\hline 56 & ITIDYSTCMGCGMCGRIC--SDSQLVGA-----FKAS-APKQPPQH--- \\
\hline 38 & IQIDYQKCIGCQMCAKTC--TDSQNFNI-----FKIS-APKTKPF---- \\
\hline 35 & NCCFRAC--IDLQGIGA-----LN-------ENDHT-- \\
\hline & JCVRMC--AEVQGIGA-----INFIN-RGSNSV---- \\
\hline WP_036838: & MVRDMTKCIRCQRCVTVC--RNYQEVDA-----LVVTG-TGLDTA---- \\
\hline $\mathrm{CDB} 53277$ & IVLDASKCIVCGRCVEVC--QNQQNVWA-----LSYLN-RGLATR---- \\
\hline JAC 89001 & VVRDMNKCVLCTRCIRAC--SQVQGMHI-----LGMIG-RGAEEEVS-- \\
\hline ABU95425 & IFYDEEKCIKCTRCIRMC--SKVQGMNV-----LSMLK-RGSEEH---- \\
\hline EFC41522 & IQIDPDKCIRCTRCIRTC--TFIQERDA-----LSMSG-RGHNES---- \\
\hline & IRLDTSKCVLCGRCIRAC--EEVAGTSA-----IIFGN-RAKKMR---- \\
\hline & IS IDREKCIKCGRCVEVC--QDVQKMNV-----LGW-FSRGRERH---- \\
\hline 3LX4_A & \\
\hline
\end{tabular}

$a a$
101
161
176
156
164
161
162
176
176
36
50
50
54
52
55
59
175
178
196
173
252
252
319
188
65
7

Organism

Magnetospirillum HybA Desulfotalea psychrophila proteobacterium CAG : 495 Acetobacter CAG:267

Paratrimastix 3

Entamoeba invadens 1

Entamoeba histolitica Acetobacter CAG:977 Azospirillum CAG:239 1

Rhodospirillum rubrum Entamoeba invadens 2

Retortamonas 1

Giardia intestinalis Trepomonas sp. PC1 2

Spironucleus 1

Spironucleus barkhanus

Pseudotrichonympha

Acetobacter CAG:977 2

Pleomorpho oryzae

Azospirillum CAG:239 2

Acanthamoeba

Sawyeria 1

Naegleria gruberi

Trichomonas 1

Monoraphidium

[FeFe]-hydrogenase outgroup

type $\mathrm{A}$

type A

type A

type A

type $\mathrm{A}$

type A

type A

type A

Nar1 precursor

type M2

type M2

type M2

type M2

type $M 2$

type M2

ype M2

type M2

type M3

type M3

type M3

type $M 3$

type M3

type M3

type M3

type M3

type M3

type M1

Fig. 1 a The modular structure of various types of [FeFe]-hydrogenase is represented by different graphical symbols of the various domains labelled according to the CDD classification [50]. Cluster N5, which has a histidine ligand [31], is coloured in red to distinguish it from standard 4Fe4S clusters. The cartoon follows the nomenclature of Meyer [12] except for type A hydrogenase, which includes previous type M3a [12] and 6/8C subtypes [15]. The colour intensity of the Small Subunit (SSU) domain at the C-terminus [41] reflects the degree of conservation (Evalue) of the recognised domain [50]. b The N-terminal parts of various of various [FeFe]-hydrogenases were aligned first by using the COBALT feature of blast searches and then manually refined by giving maximal weight to the conservation of cysteine residues that could ligate FeS clusters [32]. The cysteine motifs binding FeS clusters are highlighted in yellow. The $\delta$ proteobacterium Desulfotalea is in light blue while a proteobacterial taxa are in bold blue; eukaryotic taxa are in bold 
maturases (Table 1) have one or more genes for type M3 [FeFe]-hydrogenase, which lie in intermediate branches of the phylogenetic tree of this common type of protein, often in sister position with respect to the monophyletic clade containing the eukaryotic homologues (see Additional file 1: Figure S2A). Conversely, uncultivated Acetobacter CAG:977 and Azospirillum sp. CAG:239 have not only type M3 [FeFe]-hydrogenases, but also another type of the enzyme which we have labelled type A (Fig. 1 and Table 1).

Type A [FeFe]-hydrogenase lacks the SSU domain at the $\mathrm{C}$-terminus and characteristically shows an additional FeS cluster in the Hybrid Cluster Protein - like domain (HCP_like) at the N-terminus of the protein (Fig. 1). This structural feature derives from multicluster ferredoxins which are unrelated to type M3 [FeFe]-hydrogenases and therefore constitutes a major difference between [FeFe]-hydrogenases, making type A [FeFe]-hydrogenase a distinct enzyme (Table 1 and Fig. 2). A few $\delta$ proteobacteria such as Desulfotalea psychrophila possess ancestral versions of type A [FeFe]-hydrogenase, which is present also in the following eukaryotes: Mastigamoeba [18], Entamoeba [42] and Paratrimastix (formerly Trimastix [43]). In partial agreement with earlier reports [13, 18, 35], type A proteins occupy ancestral branches in phylogenetic trees of [FeFe]-hydrogenases, intermixing with related proteins from $\alpha$ proteobacterial organisms of metagenomic origin (Fig. 2, cf. Additional file 1: Figure S2B). Hence, we have found uncultivated $\alpha$ proteobacteria such as Acetobacter CAG:977 that possess both major types of the $[\mathrm{FeFe}]$-hydrogenases that are present in eukaryotes, namely type A and type M3 (Table 1 ). Besides a few $\delta$ proteobacteria [12] and Clostridiales [15], this combination appears to occur in the eukaryotes, Mastigamoeba [18] and Entamoeba [42], which have cytosolic forms of the hydrogenase enzymes but have lost one or more maturases as in the truly amitochondriate Monocercomonoides [26] (cf. Table 1), a situation which is likely to derive from extreme metabolic simplification following loss of MRO [19].

In the course of this phylogenetic analysis, we have additionally found consistent clustering of the most divergent type of $[\mathrm{FeFe}]$-hydrogenases, the Nar1 proteins [23], with the ancestral derivative of type M3 present in R. rubrum (Fig. 2 cf. Additional file 1: Figure S2B). This pattern could be discerned with strong bootstrap support after accurate sequence alignment of phylogenetically diverse proteins that revealed conserved elements shared with type M3 proteins from $\alpha$ proteobacteria (Fig. 2). Of note, Nar1 proteins were previously considered to form the basal branch in phylogenetic trees of [FeFe]-hydrogenases [17], presumably due to reliance on computer-assisted rather than manually cured sequence alignments for building such trees.

\section{Conclusions}

Contrary to established views that the anaerobic metabolism of eukaryotes might have been inherited through LGT phenomena from prokaryotes other than $\alpha$ proteobacteria $[11,12,17,18]$, we have shown here that a subset of $\alpha$ proteobacterial organisms of the Rhodospirillales order contain not only the two principal forms of eukaryotic [FeFe]-hydrogenases characteristic of this metabolism, but also the maturases required for the assembly of such enzymes (Table 1, Fig. 2 and Additional file 1: Figure S1). Our results therefore sustain the possibility that a single, metabolically versatile $\alpha$ proteobacterial organism might have been the ancestor of both the aerobic and anaerobic metabolism that is found in present day eukaryotes, in agreement with previous hypotheses [1, 3, 8, 44]. This simplifies the scenario of eukaryogenesis, since LGT events from organisms other than $\alpha$ proteobacteria $[9,11$, $12,18,19]$ are no longer required. On the basis of our findings, we propose a model for the molecular evolution of [FeFe]-hydrogenases and the transmission of the associated anaerobic metabolism from an ancestral organism related to current Rhodospirillales to the proto-eukaryotic cell (Fig. 3). We are currently improving the metagenomic coverage of the organisms presented here and the isolation of individual cells for single cell sequencing to complete their genomes. Once the complete genome of Acetobacter CAG:977 will be obtained and analysed in depth, we will be able to further speculate on its relationships with the possible bacterial ancestor of both the aerobic and anaerobic metabolism of eukaryotes. In any case, it now appears to be clear that this ancestor was related to present day Rhodospirillales organisms.

\section{Methods}

We have searched the latest version of the NCBI websites for proteins and genes in the NR and metagenomic databases using DeltaBLAST [45], as previously described $[32,46]$. Sequence analysis was undertaken by first constructing refined alignments of phylogenetically wide selections of proteins, in some cases combining together proteins belonging to closely related families such as the SAM maturases (see Additional file 1: Figure S1) $[10,32,33]$. Initial sequence alignments were obtained with either MUSCLE or CLUSTALW by routinely using the BLOSUM62 substitution scale and then manually implemented by considering known 3D structures of representative proteins of the orthologous family. See the legend of Fig. 2 and see Additional file 1 for specific details. Such alignments were used to build reference trees with the MEGA 5.2 program [47] and subsequently refined using known domains and protein motifs from available 3D structures and iteratively implemented until the bootstrap values of nodes containing highly divergent proteins no longer increased. Phylogenetic analysis was 


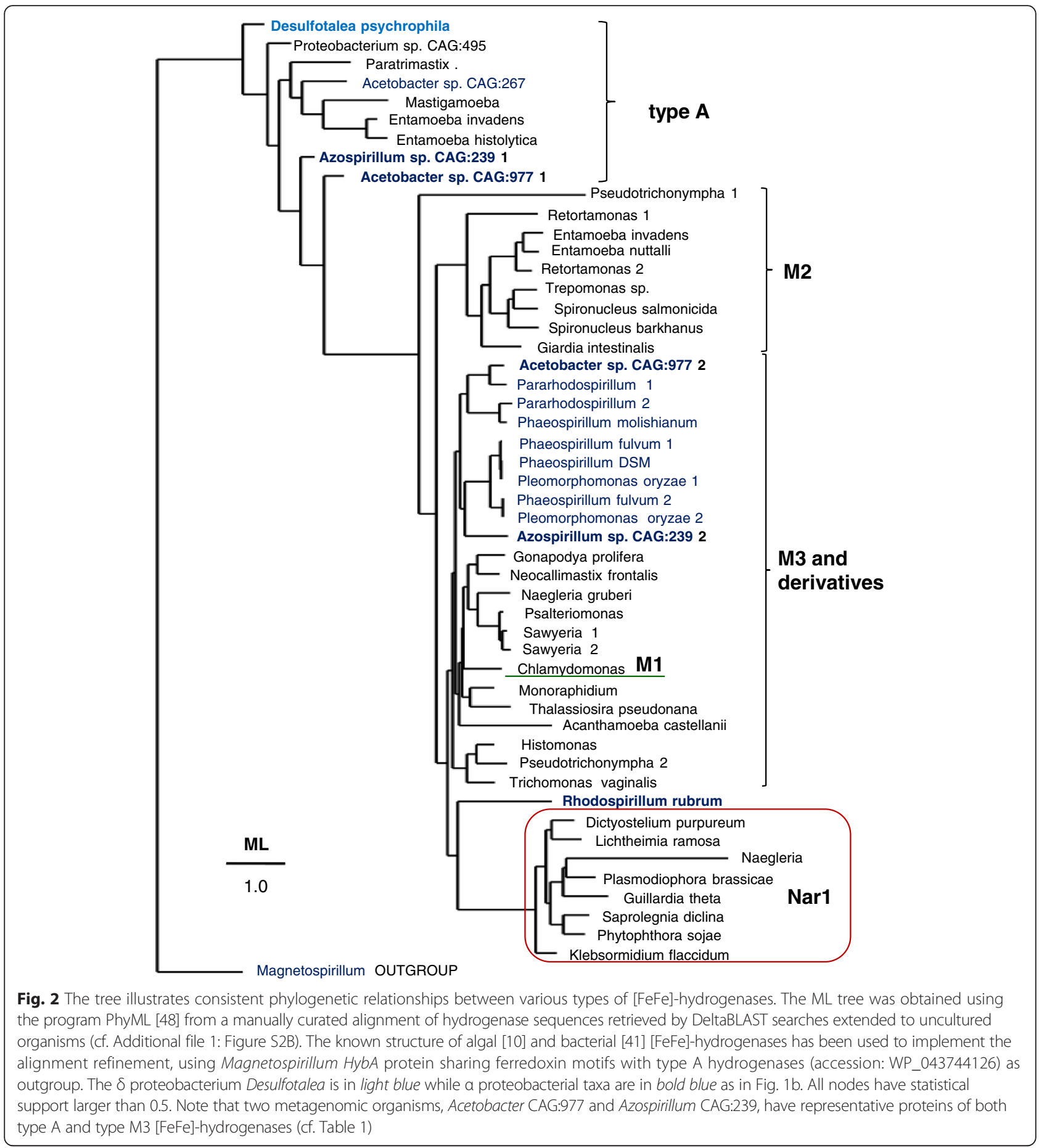

then undertaken using, in general, the PhyML program [48] with the Maximal Likelihood (ML) approach and quantitative estimation of node strength as described [49]. Additional analysis was carried out with NeighbourJoining (NJ) trees produced with the MEGA 5.2 program or the routine built into blast searches. Analysis of metagenomic data from human gut samples was undertaken as previously described [20].

\section{Reviewers' comment}

Reviewer 1: William Martin

Reviewer summary

This is a very interesting paper reporting Fe-Fe hydrogenases from alphaproteobacterial genomes that branch as sisters to eukaryotic sequences. This further supports the view that the mitochondrial ancestor was a facultative anaerobe that was capable of mitochondrial type respiration 


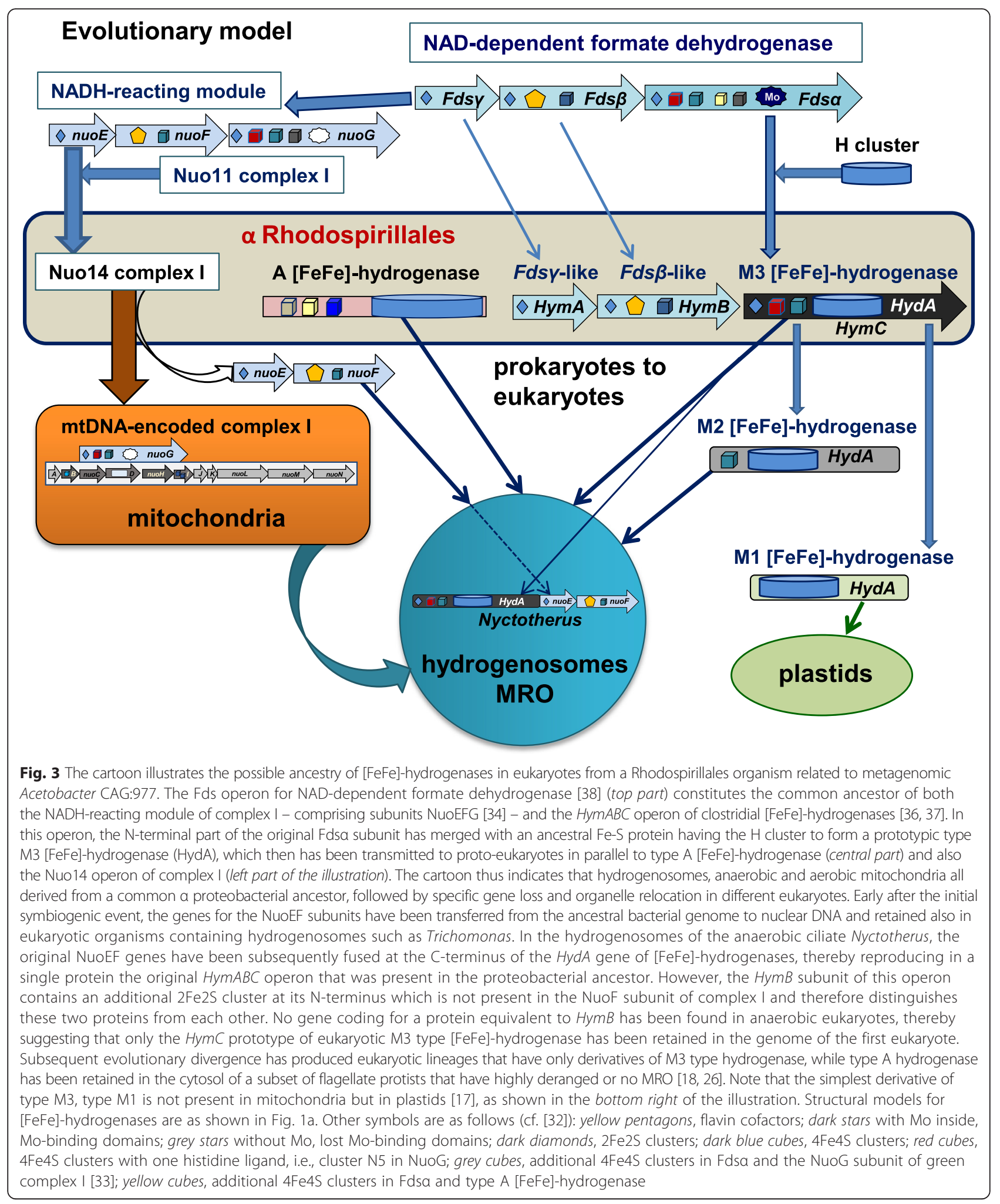

and hydrogenosomal type fermentations. People will reinvestigate the topologies, but the findings reported here are of substantial importance for understanding the evolution of eukaryote physiology. The paper should be published as is.
Reviewer recommendations to authors

I don't see a lot that needs to be done. If people use different models with this data they will get different trees, probably, but the overall message sent by the paper is 
clear: gene diversity, gene transfer and pangenomes in prokaryotes are at least as important in the eukaryote gene origin issue as hair-splitting and often highly overparameterized single gene phylogenies. There was a nice paper by $\mathrm{Ku}$ et al. in PNAS last year talking about how pangenomes figure into the eukaryote gene origin issue. There was also a nice paper by $\mathrm{Ku}$ et al. in Nature last year that bears on these issues. Ettema and Gabaldon, the recent Nature papers cited the authors here, apparently still do not understand how LGT affects eukaryote gene origin issues. It is indeed complicated, as Martin Embley and I pointed out in 1998 (Nature) ans as I explained in some detail in 1999 (BioEssays). It is gratifying that microbiologists who understand some physiology, like Mauro Degli Esposti and colleagues, understand the issues that affect the origin of eukaryotic genes from organelle ancestors that live in a world of mosaic prokaryotic chromosomes.

Minor issues

The other referees will surely have a lot to complain about, I am very happy to see these findings.

MDE et al. response: We thank the Reviewer for the very positive comments. In the revised manuscript, we have added the noted reference of Ku et al., Nature 2015 (new Ref. [8]).

\section{Reviewer 2: Nick Lane}

Reviewer summary

This is a good paper and seems to have been well done. I am not a phylogeneticist so I will defer review of the specific trees to other referees. However, the major conclusions are important, and the paper deserves publication, albeit with some recommended changes and clarifications.

This metagenomic study shows that genes for the two major eukaryotic types of [FeFe] hydrogenase are all present, along with genes for the required maturase enzymes, in the genomes of various alpha-proteobacteria related to Rhodospirillum rubrum. This finding is important in relation to the origin of the eukaryotic cell: was the bacterium that became the mitochondria a facultative anaerobe (in which case it was the ancestor of both hydrogenosomes and aerobic mitochondria) or was it an aerobic bacterium, in which case the [FeFe] hydrogenases were acquired independently on multiple occasions by lateral gene transfer from strictly anaerobic bacteria such as Clostridium? While there are several independent reasons to infer a single acquisition (see below), one problem in the past has been that not all the genes for $[\mathrm{FeFe}]$ hydrogenases and the maturases are present in alpha-proteobacteria.

This paper shows that in fact all the required genes are indeed present in close relatives of the facultatively anaerobic bacterium Rhodospirillum rubrum, and hence there is most likely to have been a common origin for aerobic and anaerobic metabolism in the mitochondria and hydrogenosomes. This is a pleasing finding that clarifies a controversial area and is wholly consistent with the predictions of the hydrogen hypothesis (Martin and Muller, Nature 1998), which explicitly predicts that the genes required for hydrogenesis derive from a facultatively anaerobic bacterium that was the common ancestor of both mitochondria and hydrogenosomes. This is also pleasing in that it is entirely consistent with bioenergetic considerations (e.g., Lane and Martin, Nature 2010) which also point to a singular origin of the eukaryotic cell in an endosymbiosis between an archaeon and a bacterium related to modern alpha-proteobacteria. Thus the paper supports the simple hypothesis that eukaryotes arose in a singular endosymbiosis between an archaeaon and a facultatively anaerobic bacterium.

Reviewer recommendations to authors

The major problem that I have with this paper relates to the evolutionary context. In my view the simple phrase 'mitochondria derive from alpha-proteobacteria' is not strictly correct, and could be seriously misleading. The problem is lateral gene transfer, not from bacteria into eukaryotes, but within bacteria (or between bacteria and archaea). The alpha-proteobacteria is a modern group, and they are well known to have evolved mechanisms that drive LGT. Given that the acquisition of mitochondria by an archaeal host cell occurred perhaps 1.5-2 billion years ago, it is not credible to talk about alpha-proteobacteria as if that modern group existed back then almost as it does now. And indeed from Fig. 2 it looks as if there has been some LGT of FeFE hydrogenase genes between different groups of bacteria.

We would therefore predict that many genes that were acquired with the ancestors of mitochondria should NOT branch with the modern alpha-proteobacteria, but rather with an assortment of modern groups. If they had all been acquired from a single endosymbiont then the key prediction is that they should all branch similarly from the base of the eukaryotes, not that they should all be found in modern alpha-proteobacteria. This seems to be the case (e.g., see Ku et al. PNAS 2015 and Ku et al. Nature 2015), although again this is by no means universally agreed. This context is entirely missing from the paper - the emphasis is wholly on the idea that genes for anaerobic metabolism should be found within modern alpha-proteobacteria, and a failure to find them would disprove a singular origin. That is not true. So while it is pleasing to find that they they are in fact common in the alpha-proteobacteria, this should not be couched as a make-or-break finding in the way that it is at several points in the paper.

I also think that Fig. 3 gives a misleading view of the hypothesis presented, and that the claim in the Conclusions 
for 'a new evolutionary model for [FeFe] hydrogenases...' is not correct and should refer instead far more explicitly to the hydrogen hypothesis, which predicted essentially all of this nearly two decades ago. My specific issue with Fig. 3 is that it makes it look as if the A [FeFe] hydrogenase and M3 [FeFe] hydrogenase were acquired separately in hydrogenosomes rather than via a mitochondrial ancestor which was similar to the alpha Rhodospiralles. The figure should make it clear that the first mitochondria were probably facultatively anaerobic with the same genes as the free-living bacteria, and that hydrogenosomes, anaerobic and aerobic mitochondria all derived from that common ancestor by specific gene loss within particular environments. This does not come across to me from that figure.

Minor issues

I have a few minor issues with some of the citations. For example in the first paragraph of the Background the statement 'originated from a symbiogenic event between an archaea and a metabolically versatile bacterium' is referenced to Sprang et al. and Williams et al. While both these references are appropriate for identifying the host cell as an archaeon, neither paper has anything specific to say about the endosymbiont being 'a metabolically versatile bacterium'. On the contrary - Sprang et al., by implying a phagocytic origin of mitochondria, actually obscures the nature of syntrophy in the endosymbiosis. The authors should cite here the hydrogen hypothesis (Martin and Muller Nature 1998); and on the nature of the host cell should also cite Sousa et al. Nature Microbiol 2016, which shows that Loki is hydrogen-dependent, also as predicted by the hydrogen hypothesis, and consistent with the results presented here.

Finally, as noted above, the paper omits any mention of other approaches to the origin of the eukaryotic cell, for example bioenergetic constraints (see e.g., Lane and Martin Nature 2010, and Lane Cold Spr Harb Pesp Biol 2014 for a discussion). These ideas have been critiqued by Booth and Doolittle PNAS 2015, and Lynch and Marinov PNAS 2015, and answered by Lane and Martin in PNAS 2015 and 2016. Other arguments in favour of a single origin of anaerobic and aerobic metabolism in mitochondria and hydrogenosomes include the very limited and repetitive metabolic repertoire of eukaryotes (equivalent to a single metabolically versatile bacterium; see e.g., Muller et al. Microbiol Moil Biol Rev 2012) and the ocean conditions during the Proterozoic when eukaryotes first arose (see e.g., various papers by Shields, Knoll and Canfield). The point is that there is a broader context than phylogenetics to framing the origin of the eukaryotic cell, and some of this literature should at least be mentioned.

That's all. I repeat, this is a good and interesting paper that is certainly worth publishing, but it would benefit from a broader discussion of the evolutionary context.
MDE et al. response: We thank the Reviewer 2 for the positive comments and constructive criticisms. Our response is as follows.

Regarding further discussion on the broad evolutionary implications of our work, we now state at the end of the revised manuscript that ...once the complete genome of Acetobacter CAG:977 will be obtained and analysed in depth, we will be able to further speculate on its relationships with the possible bacterial ancestor of both the aerobic and anaerobic metabolism of eukaryotes. In any case, it now appears to be clear that this ancestor was related to present day Rhodospirillales organisms...

With regard to the relationship between the ancestor of mitochondria and present day alpha proteobacteria, we appreciate the point raised by the Reviewer, which one of us has discussed amply in a paper that is about to be published (and available online - Degli Esposti M. Late Mitochondrial Acquisition, Really? Genome Biol Evol. 2016 Jul 3;8(6):2031-5. doi: 10.1093/gbe/evw130). However, the central problem that our paper is addressing is that previously [FeFe]-hydrogenases and their maturases were considered to be absent, or non functional, in alpha proteobacterial organisms, from which the ancestors of mitochondria have originated - according to the general consensus on the subject. For this reason we think it appropriate to maintain the relatively strong statements regarding the alpha proteobacterial ancestry of mitochondria, citing new references such as the suggested Ku et al. (Nature 2015) to further address the issue. With regard to Fig. 3, we have changed its presentation in the text as a model for the molecular evolution of [FeFe] hydrogenase, modified its graphical layout and changed its legend to better clarify that a single ancestral alpha proteobacterial organism might have transmitted both forms of hydrogenase to the protoeukaryotic cell, as suggested by the Reviewer.

\section{Additional file}

Additional file 1: Figure S1. shows the phylogenetic tree of SAM maturases HydE and HydF, while Figure S2 shows the phylogenetic tree of type M3 [FeFe]-hydrogenase (A) and of all types of [FeFe]-hydrogenases. (PDF $415 \mathrm{~kb}$ )

\begin{abstract}
Abbreviations
HCP_like, Hybrid Cluster Protein - like domain; LGT, lateral gene transfer; ML, maximum likelihood; MRO, Mitochondria Related Organelles; NJ, neighbourjoining; PFO, Pyruvate:Ferredoxin Oxidoreductase; SSU, Small Subunit
\end{abstract}

Acknowledgements

We thank Dr. Paola Bonfante (University of Turin, Italy) for discussion.

\section{Funding}

This work was sponsored by intramural funds at IIT and by CONACYT grant No. 263876 for the sabbatical research period of MDE in Mexico. 


\section{Availability of data and materials}

Supporting data are available in the online supplement Additional file 1 and also upon request.

\section{Authors' contributions}

MDE conceived the study and performed all analyses. LL performed the phylogentic analyses. MDE and EMR designed the study. MDE, DC, SR, HBN helped to draft the manuscript and DC refined the final text. All authors read and approved the final manuscript.

\section{Authors' information}

Mauro Degli Esposti is Senior Scientist at IIT, via Morego 30, 16136 Genoa, Italy - corresponding Author.

Diego Cortez, Luis Lozano and Esperanza Martinez Romero are members of the academic staff at the Centro de Ciencias Genomicas, Universidad Nacional Autonoma de Mexico (UNAM), Cuernavaca, Morelos, Mexico. Simon Rasmussen and Henrik Bjørn Nielsen are staff scientists at 3Department of Systems Biology, Center for Biological Sequence Analysis, Technical University of Denmark, Kemitorvet, Building 208, 2800 Kongens Lyngby, Denmark.

\section{Competing interests}

The authors declare that they have no competing interests.

\section{Ethics approval and consent to participate}

This is not applicable to the present work.

\section{Author details}

${ }^{1}$ Italian Institute of Technology, Via Morego 30, 16136 Genoa, Italy. ${ }^{2}$ Center for Genomic Sciences, UNAM Cuernavaca, Cuernavaca, Mexico. ${ }^{3}$ Department of Systems Biology, Center for Biological Sequence Analysis, Technical University of Denmark, Kemitorvet, Building 208, 2800 Kongens Lyngby, Denmark.

Received: 9 June 2016 Accepted: 21 July 2016

Published online: 30 July 2016

\section{References}

1. Martin W, Muller M. The hydrogen hypothesis for the first eukaryote. Nature. 1998;392(6671):37-41.

2. Ettema TJ. Evolution: Mitochondria in the second act. Nature. 2016; 531(7592):39-40.3.

3. Muller M, Mentel M, van Hellemond JJ, Henze K, Woehle C, Gould SB, Yu RY, van der Giezen M, Tielens AG, Martin WF. Biochemistry and evolution of anaerobic energy metabolism in eukaryotes. Microbiol Mol Biol Rev. 2012; 76(2):444-95.

4. Degli Esposti M. Bioenergetic evolution in proteobacteria and mitochondria. Genome Biol Evol. 2014;6(12):3238-51.

5. Gray MW. Mosaic nature of the mitochondrial proteome: Implications for the origin and evolution of mitochondria. Proc Natl Acad Sci U S A. 2015; 112(33):10133-8.

6. Lane N, Martin WF, Raven JA, Allen JF. Energy, genes and evolution: introduction to an evolutionary synthesis. Philos Trans R Soc Lond B Biol Sci. 2013;368(1622):20120253

7. Lopez-Garcia P, Moreira D. Open questions on the origin of eukaryotes. Trends Ecol Evol. 2015;30(11):697-708.

8. Ku C, Nelson-Sathi S, Roettger M, Sousa FL, Lockhart PJ, Bryant D, HazkaniCovo E, Mclnerney JO, Landan G, Martin WF. Endosymbiotic origin and differential loss of eukaryotic genes. Nature. 2015;524(7566):427-32.

9. Pittis AA, Gabaldon T. Late acquisition of mitochondria by a host with chimaeric prokaryotic ancestry. Nature. 2016;531(7592):101-4.

10. Degli Esposti M, Chouaia B, Comandatore F, Crotti E, Sassera D, Lievens PM, Daffonchio D, Bandi C. Evolution of mitochondria reconstructed from the energy metabolism of living bacteria. PLoS One. 2014;9(5):e96566.

11. Dyall SD, Yan W, Delgadillo-Correa MG, Lunceford A, Loo JA, Clarke CF, Johnson PJ. Non-mitochondrial complex I proteins in a hydrogenosomal oxidoreductase complex. Nature. 2004;431(7012):1103-7.

12. Meyer J. [FeFe] hydrogenases and their evolution: a genomic perspective. Cell Mol Life Sci. 2007;64(9):1063-84.

13. Hug LA, Stechmann A, Roger AJ. Phylogenetic distributions and histories of proteins involved in anaerobic pyruvate metabolism in eukaryotes. Mol Biol Evol. 2010;27(2):311-24.
14. Voncken FG, Boxma B, van Hoek AH, Akhmanova AS, Vogels GD, Huynen M, Veenhuis $\mathrm{M}$, Hackstein JH. A hydrogenosomal [Fe]-hydrogenase from the anaerobic chytrid Neocallimastix sp. L2. Gene. 2002;284(1-2):103-12.

15. Calusinska M, Happe T, Joris B, Wilmotte A. The surprising diversity of clostridial hydrogenases: a comparative genomic perspective. Microbiology. 2010;156(Pt 6):1575-88.

16. Jerlstrom-Hultqvist J, Einarsson E, Xu F, Hjort K, Ek B, Steinhauf D, Hultenby $\mathrm{K}$, Bergquist J, Andersson JO, Svard SG. Hydrogenosomes in the diplomonad Spironucleus salmonicida. Nat Commun. 2013;4:2493.

17. Mulder DW, Boyd ES, Sarma R, Lange RK, Endrizzi JA, Broderick JB, Peters JW. Stepwise [FeFe]-hydrogenase H-cluster assembly revealed in the structure of HydA(DeltaEFG). Nature. 2010;465(7295):248-51.

18. Nyvltova E, Stairs CW, Hrdy I, Ridl J, Mach J, Paces J, Roger AJ, Tachezy J. Lateral gene transfer and gene duplication played a key role in the evolution of Mastigamoeba balamuthi hydrogenosomes. Mol Biol Evol. 2015;32(4):1039-55.

19. Stairs CW, Leger MM, Roger AJ. Diversity and origins of anaerobic metabolism in mitochondria and related organelles. Philos Trans R Soc Lond B Biol Sci. 2015:370(1678):20140326.

20. Nielsen HB, Almeida M, Juncker AS, Rasmussen S, Li J, Sunagawa S, Plichta DR, Gautier L, Pedersen AG, Le Chatelier E, et al. Identification and assembly of genomes and genetic elements in complex metagenomic samples without using reference genomes. Nat Biotechnol. 2014:32(8):822-8.

21. Duquesne K, Prima V, Ji B, Rouy Z, Medigue C, Talla E, Sturgis JN. Draft genome sequence of the purple photosynthetic bacterium Phaeospirillum molischianum DSM120, a particularly versatile bacterium. J Bacteriol. 2012; 194(13):3559-60.

22. Xie CH, Yokota A. Pleomorphomonas oryzae gen. nov., sp. nov., a nitrogenfixing bacterium isolated from paddy soil of Oryza sativa. Int J Syst Evol Microbiol. 2005:55(Pt 3):1233-7.

23. Balk J, Pierik AJ, Netz DJ, Muhlenhoff U, Lill R. The hydrogenase-like Nar1p is essential for maturation of cytosolic and nuclear iron-sulphur proteins. EMBO J. 2004;23(10):2105-15.

24. Posewitz MC, King PW, Smolinski SL, Zhang L, Seibert M, Ghirardi ML. Discovery of two novel radical S-adenosylmethionine proteins required for the assembly of an active [Fe] hydrogenase. J Biol Chem. 2004;279(24):25711-20,

25. Dinis P, Suess DL, Fox SJ, Harmer JE, Driesener RC, De La Paz L, Swartz JR, Essex JW, Britt RD, Roach PL. X-ray crystallographic and EPR spectroscopic analysis of $\mathrm{HydG}$, a maturase in [FeFe]-hydrogenase $\mathrm{H}$-cluster assembly. Proc Natl Acad Sci U S A. 2015;112(5):1362-7.

26. Karnkowska A, Vacek V, Zubáčová Z, Treitli SC, Petrželková R, Eme L, Roger AJ, Hampl V. Eukaryote without a mitochondrial organelle. Cur Biol. 2016; 26(10):1274-84

27. Mulder DW, Shepard EM, Meuser JE, Joshi N, King PW, Posewitz MC, Broderick JB, Peters JW. Insights into [FeFe]-hydrogenase structure, mechanism, and maturation. Structure. 2011;19(8):1038-52.

28. Duquesne K, Sturgis JN. Shotgun genome sequence of the large purple photosynthetic bacterium Rhodospirillum photometricum DSM122. J Bacteriol. 2012;194(9):2380

29. Lakshmi KV, Divyasree B, Ramprasad EV, Sasikala C, Ramana CV. Reclassification of Rhodospirillum photometricum Molisch 1907, Rhodospirillum sulfurexigens Anil Kumar et al. 2008 and Rhodospirillum oryzae Lakshmi et al. 2013 in a new genus, Pararhodospirillum gen. nov., as Pararhodospirillum photometricum comb. nov., Pararhodospirillum sulfurexigens comb. nov. and Pararhodospirillum oryzae comb. nov., respectively, and emended description of the genus Rhodospirillum. Int J Syst Evol Microbiol. 2014;64(Pt 4):1154-9.

30. Madhaiyan M, Jin TY, Roy JJ, Kim SJ, Weon HY, Kwon SW, Ji L. Pleomorphomonas diazotrophica sp. nov., an endophytic N-fixing bacterium isolated from root tissue of Jatropha curcas L. Int J Syst Evol Microbiol. 2013;63(Pt 7):2477-83.

31. Baradaran R, Berrisford JM, Minhas GS, Sazanov LA. Crystal structure of the entire respiratory complex I. Nature. 2013;494(7438):443-8.

32. Degli Esposti M. Genome analysis of structure-function relationships in respiratory complex I, an ancient bioenergetic enzyme. Genome Biol Evol. 2016;8(1):126-47.

33. Degli Esposti M, Martinez Romero E. A survey of the energy metabolism of nodulating symbionts reveals a new form of respiratory complex I. FEMS Microbiol Ecol. 2016;92(6). pii: fiw084.

34. Sazanov LA. A giant molecular proton pump: structure and mechanism of respiratory complex I. Nat Rev Mol Cell Biol. 2015;16(6):375-88. 
35. Barbera' MJ, Ruiz-Trillo I, Tufts JY, Bery A, Silberman JD, Roger AJ. Sawyeria marylandensis (Heterolobosea) has a hydrogenosome with novel metabolic properties. Eukaryot Cell. 2010;9(12):1913-24.

36. Graentzdoerffer A, Rauh D, Pich A, Andreesen JR. Molecular and biochemical characterization of two tungsten- and selenium-containing formate dehydrogenases from Eubacterium acidaminophilum that are associated with components of an iron-only hydrogenase. Arch Microbiol. 2003;179(2):116-30.

37. Wang S, Huang H, Kahnt J, Mueller AP, Kopke M, Thauer RK. NADP-specific electron-bifurcating [FeFe]-hydrogenase in a functional complex with formate dehydrogenase in Clostridium autoethanogenum grown on CO. Bacteriol. 2013;195(19):4373-86

38. Oh Jl, Bowien B. Structural analysis of the fds operon encoding the NAD +-linked formate dehydrogenase of Ralstonia eutropha. J Biol Chem. 1998; 273(41):26349-60

39. Boxma B, de Graaf RM, van der Staay GW, van Alen TA, Ricard G, Gabaldon T, van Hoek AH, Moon-van der Staay SY, Koopman WJ, van Hellemond JJ, et al. An anaerobic mitochondrion that produces hydrogen. Nature. 2005; 434(7029):74-9.

40. Winkler M, Esselborn J, Happe T. Molecular basis of [FeFe]-hydrogenase function: an insight into the complex interplay between protein and catalytic cofactor. Biochim Biophys Acta. 2013;1827(8-9):974-85.

41. Nicolet Y, Piras C, Legrand P, Hatchikian CE, Fontecilla-Camps JC. Desulfovibrio desulfuricans iron hydrogenase: the structure shows unusual coordination to an active site Fe binuclear center. Structure. 1999;7(1):13-23.

42. Nixon JE, Field J, McArthur AG, Sogin ML, Yarlett N, Loftus BJ, Samuelson J. Iron-dependent hydrogenases of Entamoeba histolytica and Giardia lamblia: activity of the recombinant entamoebic enzyme and evidence for lateral gene transfer. Biol Bull. 2003;204(1):1-9.

43. Hampl V, Silberman JD, Stechmann A, Diaz-Trivino S, Johnson PJ, Roger AJ. Genetic evidence for a mitochondriate ancestry in the 'amitochondriate' flagellate Trimastix pyriformis. PLoS One. 2008;3(1):e1383.

44. Lane N, Martin W. The energetics of genome complexity. Nature. 2010; 467(7318):929-34.

45. Boratyn GM, Schaffer AA, Agarwala R, Altschul SF, Lipman DJ, Madden TL. Domain enhanced lookup time accelerated BLAST. Biol Direct. 2012;7:12.

46. Degli Esposti M, Rosas-Perez T, Servin-Garciduenas LE, Bolanos LM Rosenblueth M, Martinez-Romero E. Molecular evolution of cytochrome bd oxidases across proteobacterial genomes. Genome Biol Evol. 2015;7(3):801-20.

47. Tamura K, Peterson D, Peterson N, Stecher G, Nei M, Kumar S. MEGA5: molecular evolutionary genetics analysis using maximum likelihood, evolutionary distance, and maximum parsimony methods. Mol Biol Evol. 2011;28(10):2731-9.

48. Guindon S, Gascuel O. A simple, fast, and accurate algorithm to estimate large phylogenies by maximum likelihood. Syst Biol. 2003;52(5):696-704.

49. Anisimova M, Gascuel O. Approximate likelihood-ratio test for branches: a fast, accurate, and powerful alternative. Syst Biol. 2006;55(4):539-52.

50. Marchler-Bauer A, Derbyshire MK, Gonzales NR, Lu S, Chitsaz F, Geer LY, Geer RC, He J, Gwadz M, Hurwitz DI, et al. CDD: NCBI's conserved domain database. Nucleic Acids Res. 2015:43(Database issue):D222-226.

\section{Submit your next manuscript to BioMed Central and we will help you at every step:}

- We accept pre-submission inquiries

- Our selector tool helps you to find the most relevant journal

- We provide round the clock customer support

- Convenient online submission

- Thorough peer review

- Inclusion in PubMed and all major indexing services

- Maximum visibility for your research

Submit your manuscript at www.biomedcentral.com/submit 